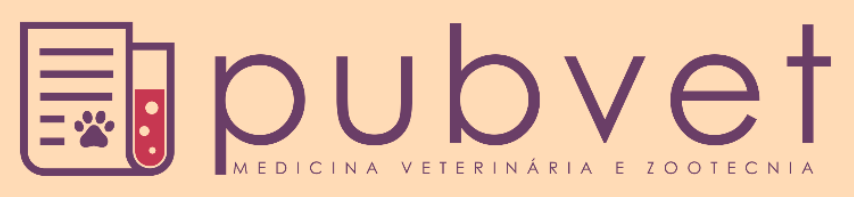

https://doi.org/10.31533/pubvet.v14n6a590.1-9

\title{
Utilização da escala composta Glasgow short form para avaliação de dois protocolos de analgesia pós-cirúrgica em cadelas submetidas à mastectomia
}

\author{
Marcelo Paulino Borges ${ }^{1^{*}}$, Maria Cecília de Lima Rorig ${ }^{2} \bullet$, Solimar Dutra da Silveira ${ }^{3 \bullet}$, \\ Thalia Vitoria Mariussi ${ }^{10}$
}

${ }^{I}$ Graduando de Medicina Veterinária na Pontifícia Universidade do Paraná. Toledo -PR Brasil.

${ }^{2}$ Professor da Pontifícia Universidade Católica do Paraná, Departamento de Medicina Veterinária. Toledo -PR Brasil.

${ }^{3}$ Residente em Clínica Cirúrgica de Pequenos Animais na Universidade Federal do Paraná. Palotina -PR Brasil.

*Autor para correspondência, E-mail: marcelopaulinoborges@gmail.com

Resumo. A preocupação com o tratamento da dor pós-operatória está fundamentada na prevenção de sofrimento desnecessário do paciente, além de proporcionar muitos benefícios fisiológicos. A dor pós-cirúrgica pode ser minimizada com maior facilidade quando se aplica a analgesia preemptiva, ou seja, a administração de analgésicos antes que ocorra o dano tecidual. Todavia, a associação de antinflamatórios não-esteroidais com opioides em protocolos antiálgicos é visto como uma forma de se reduzir as doses de cada medicamento e garantir uma abrangência maior sobre os mediadores inflamatórios. A Escala modificada de Glasgow (Short Form of the Glasgow Composite Pain Scale -CMPS$\mathrm{SF}$ ), permite a avaliação de seis categoriais comportamentais. Diante disso, o presente estudo tem como objetivo avaliar e comparar por meio de aplicação da Escala modificada de Glasgow a eficácia de dois protocolos de analgesia preemptiva utilizando adesivo de fentanil transdérmico associado a dois antinflamatórios não esteroidais (firocoxibe e carprofeno) para o controle de dor pós-operatória em cadelas submetidas à cirurgia de mastectomia. Sendo assim, 16 cadelas participaram da pesquisa e foram aleatoriamente divididas em dois grupos sendo que 8 fêmeas constituíram o grupo 1 e receberam analgesia preemptiva com firocoxibe associado ao adesivo de fentanil transdérmico e outras 8 fêmeas constituíram o grupo 2 recebendo analgesia preemptiva com carprofeno associado ao fentanil transdérmico. No pós-operatório as pacientes foram avaliadas por meio da aplicação da Escala Modificada de Glasgow em 5 tempos (T1, T2, T3, T15 e T24). O resgate analgésico somente foi efetuado quando a avaliação superou os 6 pontos na escala. Dentre as 16 pacientes que participaram da pesquisa, 11 delas $(68,75 \%)$ demonstraram controle adequado da dor, portanto, não houve a necessidade de resgate, 4 pacientes (25\%) necessitaram de resgate analgésico e 1 paciente $(6,25 \%)$ foi desclassificada da pesquisa. Conclui-se que não houve diferença no controle analgésico com ambos protocolos. A Escala Modificada de Glasgow é um método útil para avaliação do grau da dor em cadelas submetidas à mastectomia. $\mathrm{O}$ fentanil transdérmico demonstrou boa atuação no controle da dor simultaneamente associado a ambos antinflamatórios, porém é de suma importância que o adesivo esteja corretamente fixado à pele para adequada liberação do princípio ativo.

Palavras chave: analgesia, dor, Glasgow, mastectomia

\section{Using the Glasgow short form composite scale to evaluate two postoperative analgesia protocols in female dogs submitted to mastectomy}

Abstract. The concern with the treatment of postoperative pain is based on the prevention of unnecessary suffering of the patient, in addition to providing many physiological benefits. Post- 
surgical pain can be minimized more easily when preemptive analgesia is applied, that is, the administration of analgesics before tissue damage occurs. However, the association of nonsteroidal anti-inflammatory drugs with opioids in anti-pain protocols is seen as a way to reduce the doses of each medication and ensure a greater coverage of inflammatory mediators. The modified Glasgow Scale (Short Form of the Glasgow Composite Pain Scale -CMPS-SF), allows the assessment of six behavioral categories. In view of this, the present study aims to evaluate and compare, by applying the modified Glasgow Scale, the effectiveness of two different preemptive analgesia protocols using transdermal fentanyl patch associated with two non-steroidal anti-inflammatory drugs (firocoxib and carprofen) for the control of postoperative pain in dogs undergoing mastectomy surgery. Thus, 16 bitches participated in the research and were randomly divided into two groups with 8 females constituting the 1 group and receiving preemptive analgesia with firocoxib associated with transdermal fentanyl patch and another 8 females constituting the 2 group receiving analgesia preemptive with carprofen associated with transdermal fentanyl. Postoperatively, patients were evaluated using the Glasgow Modified Scale in 5 steps (T1, T2, T3, T15 and T24). The analgesic rescue was only performed when the assessment exceeded 6 points on the scale. Among the 16 patients who participated in the research, 11 of them $(68.75 \%)$ demonstrated adequate pain control, therefore, there was no need for rescue, 4 patients $(25 \%)$ needed analgesic rescue and 1 patient $(6.25 \%)$ was disqualified from the search. It was concluded that there was no difference in analgesic control with both protocols. The Glasgow Modified Scale is a useful method for assessing the degree of pain in bitches undergoing mastectomy. Transdermal fentanyl has demonstrated good performance in pain control simultaneously associated with both anti-inflammatory drugs, however it is of utmost importance that the patch is correctly attached to the skin for proper release of the active ingredient.

Keywords: analgesia, pain, Glasgow, mastectomy

\section{Uso de la escala compuesta de Glasgow short form para evaluar dos protocolos de analgesia posquirúrgica en perras sometidas a mastectomía}

Resumen. La preocupación con el tratamiento del dolor postoperatorio se basa en la prevención del sufrimiento innecesario del paciente, además de proporcionar muchos beneficios fisiológicos. El dolor posquirúrgico se puede minimizar más fácilmente cuando se aplica analgesia preventiva, es decir, la administración de analgésicos antes de que ocurra el daño tisular. Sin embargo, la asociación de los medicamentos antiinflamatorios no esteroideos con los opioides en los protocolos contra el dolor se ve como una forma de reducir las dosis de cada medicamento y garantizar una mayor cobertura de mediadores inflamatorios. La Escala de Glasgow (Forma abreviada de la Escala de dolor compuesta de Glasgow -CMPS-SF) modificada, permite la evaluación de seis categorías de comportamiento. En vista de esto, el presente estudio tiene como objetivo evaluar y comparar, aplicando la Escala de Glasgow modificada, la efectividad de dos protocolos de analgesia preventiva que usan parches de fentanilo transdérmicos asociados con dos medicamentos antiinflamatorios no esteroideos (firocoxib y carprofeno) para el control de dolor postoperatorio en perros sometidos a cirugía de mastectomía. Por lo tanto, 16 perras participaron en la investigación y se dividieron al azar en dos grupos con 8 hembras que constituyen el grupo $1 \mathrm{y}$ recibieron analgesia preventiva con firocoxib asociado con el parche transdérmico de fentanilo y otras 8 hembras que constituyen el grupo 2 que recibieron analgesia preventiva con carprofeno asociado con fentanilo transdérmico. En el postoperatorio, los pacientes fueron evaluados utilizando la Escala Modificada de Glasgow en 5 pasos (T1, T2, T3, T15 y T24). El rescate analgésico solo se realizó cuando la evaluación excedió los 6 puntos en la escala. Entre las 16 pacientes que participaron en la investigación, 11 de ellos (68.75\%) demostraron un control adecuado del dolor, por lo tanto, no hubo necesidad de rescate, 4 pacientes (25\%) necesitaron rescate analgésico y 1 paciente $(6.25 \%)$ fue descalificada del estudio. Se concluyó que no hubo diferencia en el control analgésico con ambos protocolos. La Escala Modificada de Glasgow es un método 
útil para evaluar el grado de dolor en perras sometidas a mastectomía. El fentanilo transdérmico demostró un buen rendimiento en el control del dolor asociado simultáneamente con ambos medicamentos antiinflamatorios, sin embargo, es de suma importancia que el parche esté correctamente adherido a la piel para la liberación adecuada del ingrediente activo.

Palabras clave: analgesia, dolor, Glasgow, mastectomía

\section{Introdução}

A preocupação com o tratamento da dor pós-operatória está fundamentada na prevenção de sofrimento desnecessário do paciente, além de proporcionar muitos benefícios fisiológicos (Fantoni et al., 2002; Rozanski \& Rush, 2009). A dor pós-cirúrgica pode ser minimizada com maior facilidade quando se aplica a analgesia preemptiva, ou seja, a administração de analgésicos antes que ocorra o dano tecidual (Maddison et al., 2011; Natalini, 2007; Paddleford, 2001).

A associação de anti-inflamatórios não-esteroidais (AINES) com opioides em protocolos antiálgicos é vista como uma forma de se reduzir as doses de cada medicamento e garantir uma abrangência maior sobre os mediadores inflamatórios (Carroll, 2012).

O firocoxibe é um anti-inflamatório não-esteroidal (AINE), inibidor seletivo da COX-2 de uso exclusivo veterinário (Booth \& McDonaldo, 1992; Spinosa et al., 2002). O carprofeno é outra opção de AINE com excelente ação anti-inflamatória, analgésica e antipirética (Andrade, 2011). Estudos relacionam seus efeitos a inibição preferencial da COX-2 (Spinosa et al., 2002).

$\mathrm{O}$ reconhecimento da dor nos animais pode ser avaliado principalmente pela observação das alterações de comportamento da espécie. A Escala modificada de Glasgow (Short Form of the Glasgow Composite Pain Scale - CMPS-SF), permite a avaliação de seis categorias comportamentais (vocalização, atenção à ferida cirúrgica, mobilidade do paciente, resposta ao toque, comportamento perante ao meio, postura e atividade), possibilitando assim graduar a intensidade da dor (Reid et al., 2007).

O presente estudo tem como objetivo avaliar e comparar por meio de aplicação da Escala modificada de Glasgow (Short Form of the Glasgow Composite Pain Scale) (Anexo 1), a eficácia de dois diferentes protocolos de analgesia preemptiva utilizando adesivo de fentanil transdérmico associado a dois antinflamatórios não esteroidais (firocoxibe e carprofeno), para o controle de dor pós-operatória em cadelas submetidas à cirurgia de mastectomia.

\section{Material e métodos}

O projeto foi desenvolvido na Clínica Veterinária Universitária - CLIVET da Pontifícia Universidade Católica do Paraná, campus Toledo e no Hospital Veterinário Aukmia, localizado na Rua Almirante Barroso, 2546, Centro de Toledo, Paraná. As atividades tiveram início logo após aprovação do Comitê de Ética no Uso de Animais - CEUA da PUCPR (Protocolo de submissão: 01215).

Durante o estudo, 16 fêmeas foram avaliadas no projeto (Figura 1), sendo que 8 pacientes participaram do grupo (G1), que recebeu analgesia preemptiva com firocoxibe na dose de $5 \mathrm{mg} / \mathrm{kg}$ (Viana, 2014), associado ao adesivo de fentanil transdérmico (concentração de 25, 50, 75 ou 100 $\mu \mathrm{g} / \mathrm{hora})$ de acordo com o peso de cada paciente $(25 \mu \mathrm{g} / \mathrm{h}$ para cães de $5 \mathrm{a} 10 \mathrm{~kg}$ e para cães entre $10 \mathrm{e}$ $20 \mathrm{~kg}, 50 \mu \mathrm{g} / \mathrm{h}$ ). Já o segundo grupo (G2), também com 8 pacientes, receberam analgesia preemptiva com carprofeno na dose de 4,4 mg/kg (Viana, 2014) associado ao fentanil transdérmico nas mesmas recomendações supracitadas (Tabela 1).

As pacientes foram internadas 24 horas prévias ao procedimento cirúrgico, iniciando a preparação cirúrgica por meio de tricotomia (Figura 2) em região cervico-torácica ou torácica e fixação do adesivo de fentanil transdérmico (DUROGESIC ${ }^{\circledR}$ D-TRANS) (Figura 3) de acordo com o peso corporal de cada paciente, como indicado por (Fantoni et al., 2002). Também foi administrado neste mesmo momento o antinflamatório não esteroidal, por via oral (Figura 4).

O protocolo anestésico foi realizado com medicação pré-anestésica a base de acepromazina na dose de $0,05 \mathrm{mg} / \mathrm{Kg}$ por via intramuscular e após 20 minutos, indução com propofol na dose de $4 \mathrm{mg} / \mathrm{kg} / \mathrm{IV}$ 
seguida de manutenção com sevoflurano (conectado a uma fonte de oxigênio a 100\%). Caso necessário, durante o período transoperatório era realizado resgate analgésico com uso de fentanil na dose de 0,02 $\mathrm{mg} / \mathrm{Kg}$ pela via endovenosa (Massone, 2011). (Figura 5).

Após o término da intervenção cirúrgica, as fêmeas eram mantidas em ambiente tranquilo (Figura 6) para a aplicação da Escala modificada de Glasgow (Short Form of the Glasgow Composite Pain Scale) (Anexo 1).

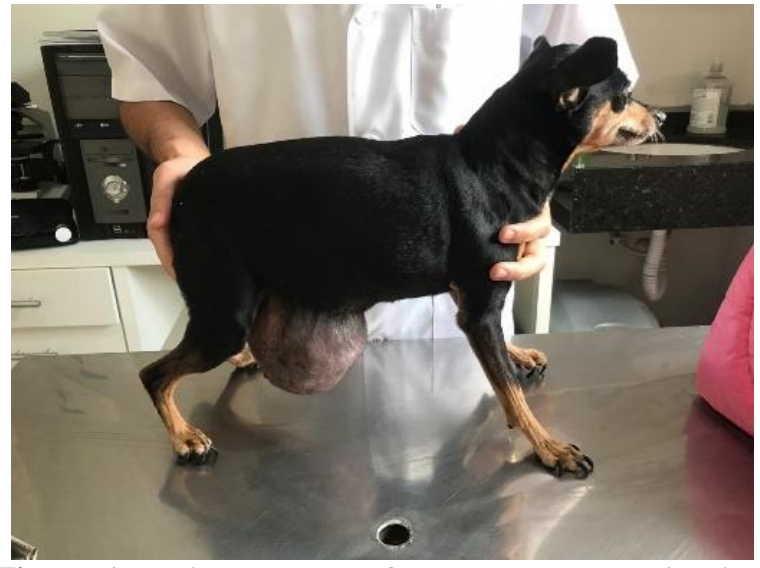

Figura 1. Paciente com neoformação em mama inguinal submetida a exame pré-operatório.

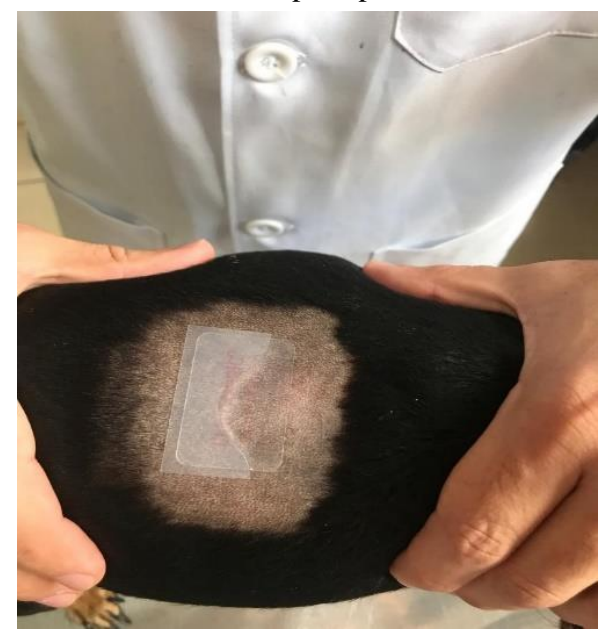

Figura 3. Adesivo de fentanil fixado à pele.

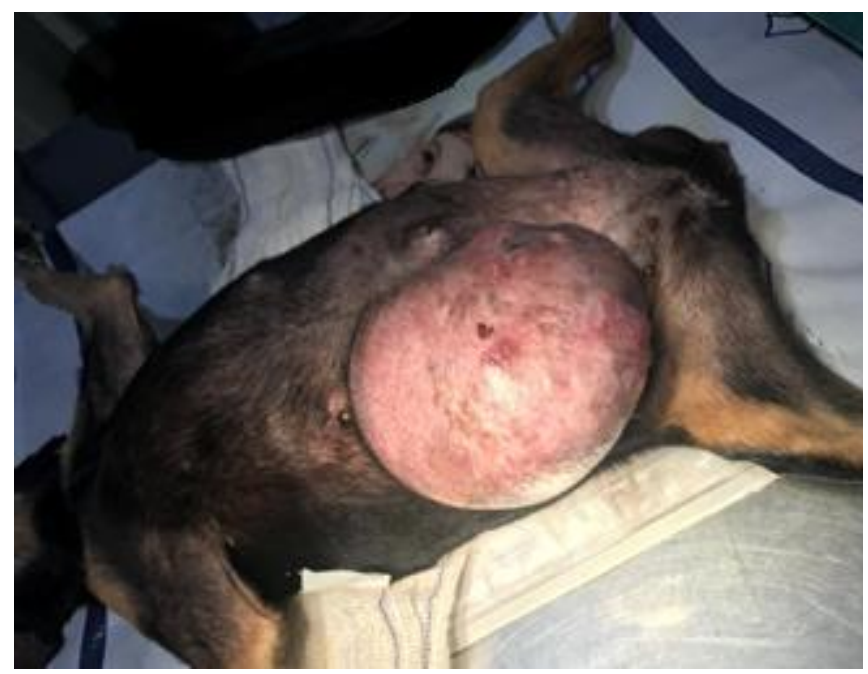

Figura 5. Paciente posicionado após indução anestésica.

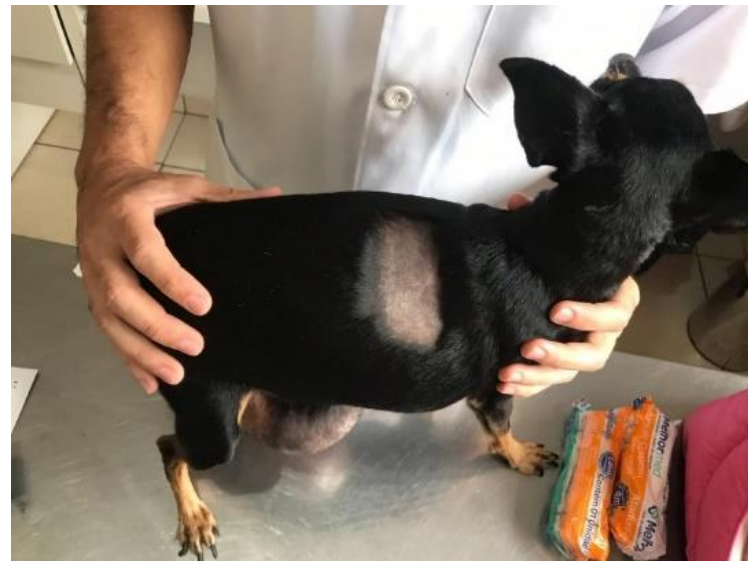

Figura 2. Imagem da área tricotomizada para fixação do adesivo.

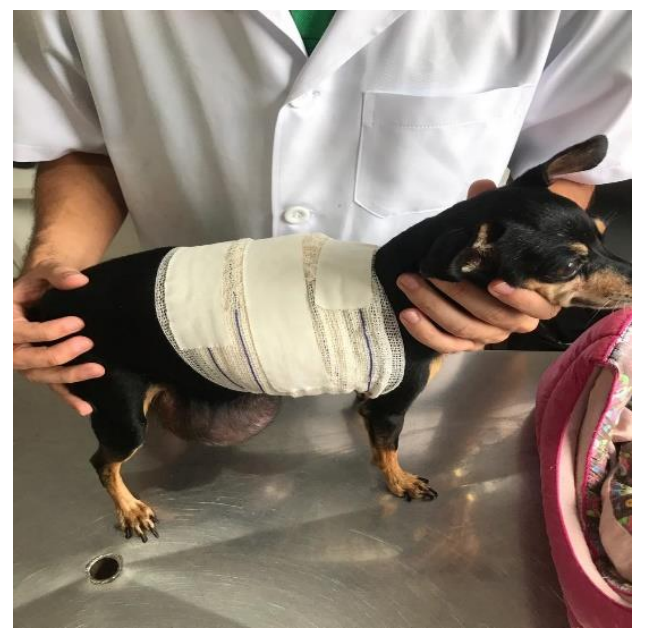

Figura 4. Finalização da fixação do adesivo e proteção com bandagem.

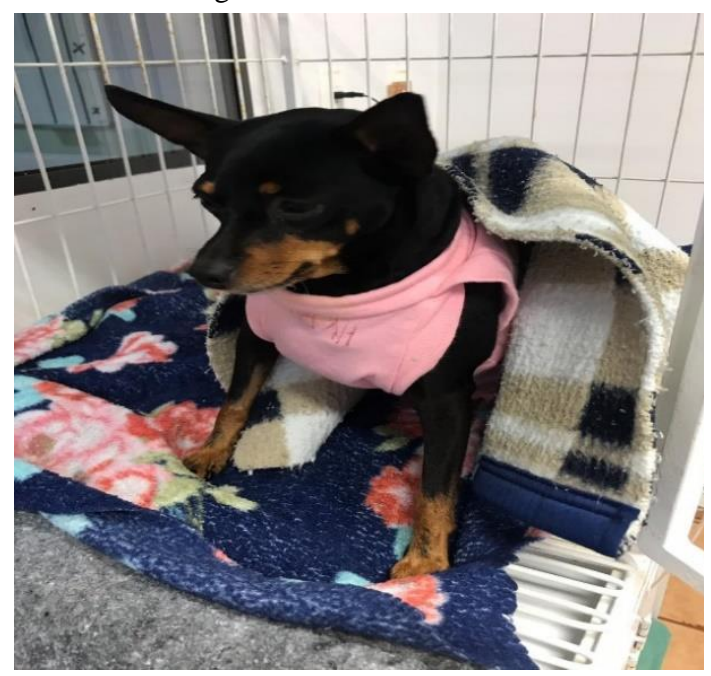

Figura 6 Paciente no pós-operatório imediato (T1). 
De acordo com a avaliação obtida na escala de dor, o resgate analgésico era realizado quando a pontuação ultrapassava 6 pontos (Reid et al., 2007), utilizando-se metadona na dose de $0,2 \mathrm{mg} / \mathrm{kg}$ por via intravenosa como forma de recuperação analgésica (Viana, 2014).

As fêmeas eram avaliadas imediatamente após a recuperação da consciência pela escala, aplicação da escala em cinco tempos (T1, T2, T3, T15 e T24). A primeira análise era realizada com uma hora após o término do procedimento (T1) (Figura 6), a segunda observação era realizada duas horas após a cirurgia (T2), a terceira após três horas decorridas da cirurgia (T3), a quarta avaliação 15 horas após (T15) e a quinta e última avaliação era realizada após 24 horas do término procedimento (T24).

Decorrida a última avaliação pela Escala Modificada de Glasgow, o adesivo de fentanil transdérmico foi removido e as pacientes receberam alta com prescrição de amoxicilina + clavulanato de potássio, dipirona sódica e continuaram com a administração do antinflamatório por mais 3 dias. Prescreveu-se tramadol a cada 12 horas durante 4 dias além de orientações sobre cuidados com a ferida cirúrgica.

Tabela 1. Número de pacientes do projeto.

\begin{tabular}{|c|c|c|c|c|c|}
\hline Pacientes & AINE & Resgate Metadona & Resgate Fentanil & Adesivo deslocado & Remoção do adesivo \\
\hline 1 & Carprofeno & Não & Sim & Não & Não \\
\hline 2 & Carprofeno & Não & Não & Não & Não \\
\hline 3 & Carprofeno & Não & Não & Não & Não \\
\hline 4 & Carprofeno & Não & Não & Não & Não \\
\hline 5 & Carprofeno & Sim & Não & Sim & Não \\
\hline 6 & Carprofeno & Não & Não & Não & Não \\
\hline 7 & Carprofeno & Não & Não & Não & Não \\
\hline 8 & Carprofeno & Não & Não & Não & Não \\
\hline 9 & Firocoxibe & Não & Não & Não & Não \\
\hline 10 & Firocoxibe & Não & Sim & Não & Não \\
\hline 11 & Firocoxibe & Sim & Não & Sim & Não \\
\hline 12 & Firocoxibe & Não & Não & Não & Não \\
\hline 13 & Firocoxibe & Não & Não & Não & Não \\
\hline 14 & Firocoxibe & Não & Não & Não & Não \\
\hline 15 & Firocoxibe & Não & Não & Não & Não \\
\hline 16 & Firocoxibe & Sim & Paciente excluído & Paciente excluído & Sim \\
\hline
\end{tabular}

\section{Resultados}

Foram atendidas 8 pacientes seguindo o protocolo utilizando firocoxibe (G1), e 8 pacientes utilizando o segundo protocolo, sendo administrado carprofeno (G2) como antinflamatório não-esteroidal (AINE).

Em 11 pacientes $(68,75 \%)$ (Gráfico 1) observou-se adequado controle da dor, menor requerimento de sevoflurano durante a cirurgia e rápido retorno anestésico. Nestes não houve a necessidade de resgate analgésico. Aproximadamente uma hora após a cirurgia as pacientes já se alimentavam, ignoravam a ferida, caminhavam normalmente e quando sofriam uma leve pressão próximo a ferida, elas se comportavam de forma indiferente. Diante dessas observações e de acordo com a Escala de Glasgow, as pacientes não apresentavam dor.

Na paciente $\mathrm{n}^{\circ} 5$ com tricotomia não realizada corretamente, devido à um defeito na lâmina da máquina, observou-se nas primeiras avaliações ausência de dor. Porém, na terceira avaliação (T3) apresentava vocalização e agressividade. Em outra paciente $\left(n^{\circ} 11\right)$ observou-se alteração nos parâmetros vitais com aumento da pontuação da escala, e ao verificar o adesivo, constatou-se que o mesmo não estava aderido à pele. Diante da avaliação, procedeu-se ao resgate analgésico com metadona, nas duas pacientes. No total do projeto somente em duas pacientes $(12,5 \%)$ houve necessidade de resgate analgésico com metadona. Nas pacientes N 1 e $10(12,5 \%)$ houve necessidade de resgate analgésico no transoperatório, por apresentarem alterações de parâmetros, como elevação das frequências respiratória (FR) e cardíaca (FC), além de dilatação de pupilas. Diante disso, administrou-se fentanil na dose de 0,02 $\mathrm{mg} / \mathrm{kg}$ por via endovenosa. As mesmas, porém, após a cirurgia não obtiveram pontuação superior a 6 
pontos na escala, não necessitando de recuperação analgésica. A paciente $\mathrm{n}^{\circ} 16(6,25 \%)$ apresentou inquietação e tremores quatro horas após a fixação do adesivo e por esse motivo foi excluída da pesquisa.

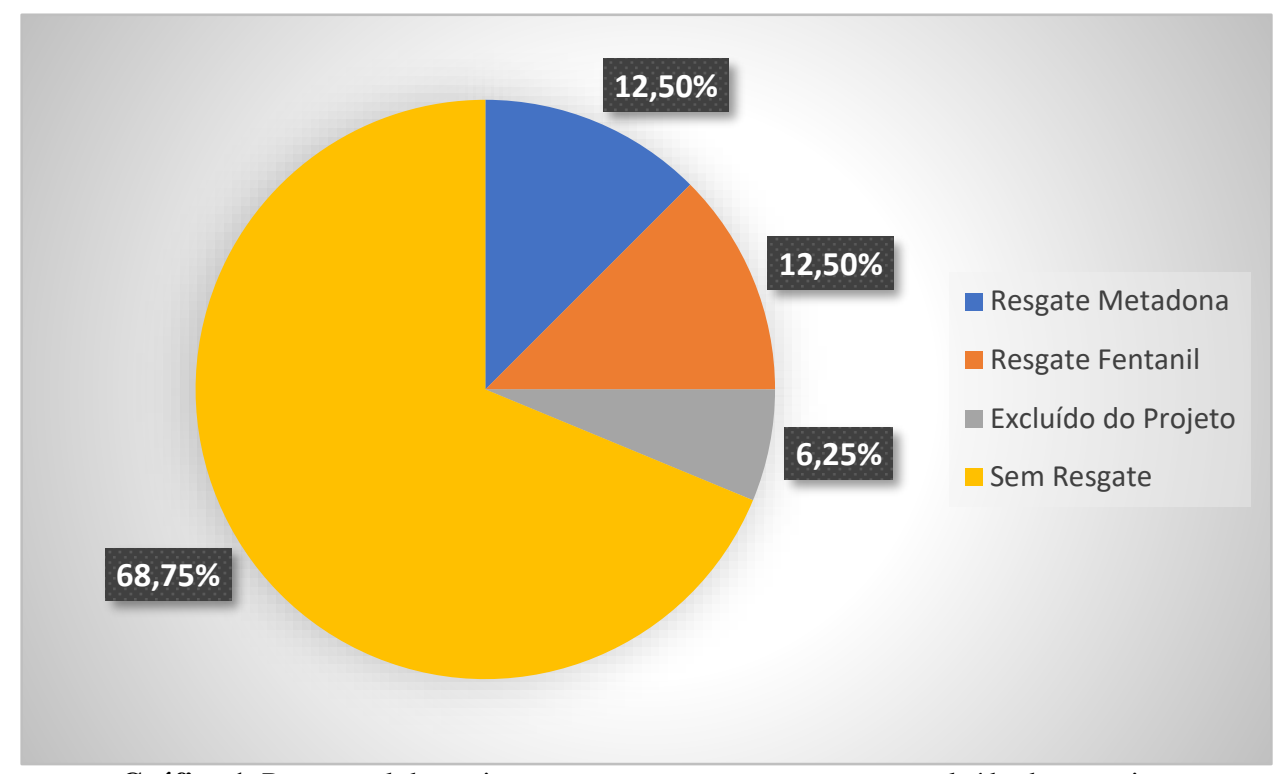

Gráfico 1. Percentual de pacientes sem resgate, com resgate e excluído da pesquisa.

\section{Discussão}

A mastectomia é um procedimento cirúrgico extenso e de ampla ressecção tecidual (Credie, 2013), que tem como consequência elevado nível de dor o que exige anestesia e analgesia adequadas (Assumpção et al., 2017). Dentro desse contexto, os resultados do presente estudo referente à avaliação analgésica pós-cirúrgica em cadelas submetidas à mastectomia revelaram que em $68,75 \%$ das pacientes não houve necessidade de resgate analgésico (pré e pós-cirúrgico), evidenciando a efetividade do fentanil transdérmico (DUROGESIC ${ }^{\circledR}$ D-TRANS) como mencionado por Marcato (2010).

Aplicou-se o adesivo 24 horas antes do procedimento cirúrgico com intuito principal de realizar adequado controle de dor, reduzir as doses dos medicamentos e efeitos colaterais no período transoperatório o que corrobora com Assumpção et al. (2017).

Um dia antes da cirurgia foi administrado firocoxibe (G1) ou carprofeno (G2), anti-inflamatórios não esteroidais (AINEs) de forma preemptiva. Também foi associado a dipirona, a qual é relatada por Fantoni et al. (2002) como tendo efeito sinérgico com AINES e opioides. Segundo a bula do DUROGESIC ${ }^{\circledR}$ D-TRANS é importante fazer correta tricotomia do local da aplicação do adesivo e posteriormente recobrir com atadura para fixação (Aleixo et al., 2004) (Figura 4). No presente estudo, duas pacientes $\left(\mathrm{N}^{\circ} 5\right.$ e 11) (12,5\%) necessitaram de resgate analgésico no pós-cirúrgico, sendo, uma delas $\left(n^{\circ} 5\right)$ pela não realização adequada da tricotomia e consequentemente o deslocamento do adesivo.

A bula do fentanil transdérmico orienta que o adesivo pode não aderir satisfatoriamente a todos os pacientes, e enfatiza que há uma necessidade de verificar com frequência se o mesmo está bem aderido à pele. Com a remoção do adesivo, as concentrações séricas diminuem de forma gradativamente, em torno de $50 \%$ em cerca de 17 horas depois de um dia de aplicação (DUROGESIC ${ }^{\circledR}$ DTRANS). Portanto, como o procedimento é doloroso e o adesivo não estava em pleno contato com à pele, as pacientes $\left(\mathrm{n}^{\circ} 5\right.$ e 11$)$ apresentaram pontuação indicativa de dor. Diante da avaliação, procedeu-se ao resgate analgésico com metadona. Aplicou-se metadona na dose de $0,2 \mathrm{mg} / \mathrm{kg}$ por via intravenosa lenta, como orientado por Viana (2014) constatando-se melhora progressiva até obter pontuação inferior a 6 pontos na escala.

As pacientes $\mathrm{n}^{\circ} 1$ e $10(12,5 \%)$ necessitaram de resgate analgésico com fentanil durante o período transoperatório. Elas apresentaram alterações de parâmetros fisiológicos (FR, FC e dilatação de pupilas). Diante dos fatos, administrou-se por via endovenosa fentanil na dose de $0,02 \mathrm{mg} / \mathrm{kg}$. As mesmas, porém, no pós-cirúrgico não apresentaram pontuação superior a 6 pontos na escala, não necessitando de recuperação analgésica o que está de acordo com Druziani (2018) em seu estudo, pois o fentanil no 
transoperatório apresenta analgesia com início rápido e de curta duração, não interferindo nas avaliações no pós-operatório.

Nos dois protocolos ( $\mathrm{G} 1$ e G2) observou-se bom controle da dor, somente houve resgate analgésico no pós-cirúrgico onde o adesivo não estava bem aderido à pele. Uma pesquisa feita por Cruz (1999) demonstrou que o carprofeno é capaz de controlar a dor pós-operatória._O carprofeno possui reduzido efeito colateral gastrintestinal (Luna et al., 2007) além de apresentar bom efeito quando administrado no pré e pós-cirúrgico, otimizando o controle da dor. Aparentemente os AINEs são ideais para serem utilizados de forma preemptiva (Camargo, 2008; Gramke et al., 2006).

De acordo com o presente estudo, o firocoxibe mostrou-se efetivo no controle da dor. A grande maioria dos anti-inflamatórios não esteroidais possui_capacidade de inibir as cicloxigenases 1 e 2 . $\mathrm{O}$ firocoxibe é um inibidor seletivo da Cox 2, diminuindo efeitos adversos no trato gastrintestinal. Também pode ser utilizado como adjuvante no tratamento de tumores mamários malignos uma vez que metade desses tumores apresenta expressão da enzima Cox 2 (Elston, 2012). A expressão desta enzima possui relação com a progressão tumoral, ressaltando a associação positiva do firocoxibe como adjuvante (Cassali et al., 2011; Lavalle et al., 2012).

A associação de fármacos analgésicos é amplamente utilizada na medicina veterinária, constituindo a base da analgesia multimodal que tem como objetivos analgesia efetiva e diminuição de possíveis efeitos colaterais (Lamond, 2008; Montes et al., 2000).

Neste estudo a escala demonstrou eficácia na avaliação da dor o que está de acordo com Comassetto et al. (2017) que afirmam que a escala de Glasgow apresenta maior sensibilidade para detectar o momento necessário para a realização do resgate analgésico, e ressaltam ainda que a inexperiência do avaliador não compromete a qualidade da avaliação.

\section{Conclusão}

Diante dos resultados obtidos nesta pesquisa, constatou-se que não houve diferença no controle analgésico com ambos protocolos avaliados. A Escala Modificada de Glasgow é um método útil para avaliação do grau da dor em cadelas submetidas à mastectomia. $\mathrm{O}$ fentanil transdérmico demonstrou boa atuação no controle da dor simultaneamente associado ao firocoxibe ou ao carprofeno. Vale salientar a grande importância da fixação do adesivo de fentanil à pele para adequada liberação do princípio ativo.

\section{Referências bibliográficas}

Aleixo, G. A. S., Ribeiro, V. M., Maciel Júnior, B. S., Sousa, A. A., \& Oliveira, C. R. (2004). Use of the analgesic fentanyl patch in a dog. Ciência Veterinária Nos Trópicos, 27(2/3), 140-144.

Andrade, S. F. (2011). Manual de terapêutica veterinária. Editora Roca.

Assumpção, A. E., Naspolini, B., Santalucia, S., Heymanns, A. C., \& Piovezan, A. P. (2017). Avaliação de dois protocolos de analgesia transoperatória em cadelas submetidas à mastectomia unilateral total. Acta Scientiae Veterinariae, 45, 1-8.

Booth, N. H., \& McDonaldo, L. E. (1992). Farmacologia e terapêutica em veterinária. Guanabara Koogan.

Camargo, J. B. (2008). Análise da eficácia da analgesia preemptiva: Revisão bibliográfica. Monografia apresentada para obtenção do título de Especialista Latu Sensu em Anestesiologia Veterinária. Brasil: Faculdade de Jaguariúna.

Carroll, G. L. (2012). Anestesia e analgesia de pequenos animais (Manole (ed.); Vol. 6460).

Cassali, G. D., Lavalle, G. E., De Nardi, A. B., Ferreira, E., Bertagnolli, A. C., Estrela-Lima, A., Alessi, A. C., Daleck, C. R., Salgado, B. S., \& Fernandes, C. G. (2011). Consensus for the diagnosis, prognosis and treatment of canine mammary tumors. Brazilian Journal of Veterinary Pathology, 4(2), 153-180.

Comassetto, F., Rosa, L., Ronchi, S. J., Fuchs, K., Regalin, B. D., Regalin, D., Padiha, V., \& Oleskovicz, N. (2017). Correlação entre as escalas analógica visual, de Glasgow, Colorado e Melbourne na avaliação de dor pós-operatória em cadelas submetidas à mastectomia total unilateral. Arquivo Brasileiro de Medicina Veterinária e Zootecnia, 69(2), 355-363. DOI: http://dx.doi.org/10.1590/1678-4162-9075 
Credie, L. F. G. A. (2013). Avaliação perioperatória da técnica de anestesia por tumescência em cadelas submetidas à mastectomia unilateral. 2013. 135p. Faculdade de Medicina de Botucatu - UNESP, Botucatu.

Cruz, M. L. (1999). Efeitos do flunixin, ketoprofeno, carprofeno, buprenorfina e placebo para analgesia pós-operatória em cães submetidos à osseossíntese de fêmur. A Hora Veterinária, Supl., 11-17.

Druziani, J. T. (2018). Avaliação do potencial analgésico do Maropitant em cadelas submetidas à ovariohisterectomia eletiva.

Elston, F. (2012). Avaliação clínica e imunoistoquímica de tumores mamários em cadelas submetidas a tratamento com inibidor da cicloxigenase-2 (firocoxibe). 2012. 68 f. Dissertação (Mestrado). Universidade Estadual Paulista, Botucatu.

Fantoni, D. T., Mastrocinque, S., Fantoni, D. T., \& Cottopassi, S. R. (2002). Fisiopatologia e controle da dor. In D. T. Fabntoni (Ed.), Anestesia em cães e gatos (pp. 323-336). Roca.

Fossum, T. W. (2014). Cirurgia de pequenos animais (4th ed., Vol. 1). Elsevier Brasil.

Gramke, H.-F., Petry, J. J. J., Durieux, M. E., Mustaki, J.-P., Vercauteren, M., Verheecke, G., \& Marcus, M. A. E. (2006). Sublingual piroxicam for postoperative analgesia: preoperative versus postoperative administration: a randomized, double-blind study. Anesthesia \& Analgesia, 102(3), 755-758. DOI: 10.1213/01.ane.0000197611.89464.98

Lamond, L. A. (2008). Multimodal management in veterinary medicine: The physiologic basis of pharmacologic therapies. Veterinary Clinic of Small Animal, 38, 1173-1186. DOI: https://doi.org/10.1016/j.cvsm.2008.06.005

Lavalle, G. E., Campos, C. B., \& Bertagnolli, A. C. (2012). Canine malignant mammary gland neoplsms with advanced clinical staging treated with carboplatin and cyclooxygenase inhibitors. In Vivo, 26, 375-380.

Luna, S. P. L., Basílio, A. C., Steagall, P. V. M., Machado, L. P., Moutinho, F. Q., Takahira, R. K., \& Brandão, C. V. S. (2007). Evaluation of adverse effects of long-term oral administration of carprofen, etodolac, flunixin meglumine, ketoprofen, and meloxicam in dogs. American Journal of Veterinary Research, 68(3), 258-264. DOI: https://doi.org/10.2460/ajvr.68.3.258

Maddison, J. E., Page, S. W., \& Church, D. B. (2011). Farmacologia clínica de pequenos animais. In G. A. Philip \& A. F. Thomas (Eds.), Medicamentos e reprodução (pp. 520-537). Elsevier Brasil.

Marcato, J. de A. (2010). Pancreatite em cães.

Massone, F. Anestesiologia Veterinária: Farmacologia e Técnicas. 6. ed. Rio de Janeiro: Guanabara Koogan, 2011.

Montes, A., Warner, W., \& Puig, M. M. (2000). Use of intravenous patient-controlled analgesia for the documentation of synergy between tramadol and metamizol. British Journal of Anaesthesia, 85(2), 217-223. DOI: https://doi.org/10.1093/bja/85.2.217

Natalini, C. C. (2007). Teoria e técnicas em anestesiologia veterinária. Srtmed.

Paddleford, R. R. (2001). Manual de anestesia em pequenos animais. Roca.

Reid, J., Nolan, A. M., Hughes, J. M. L., Lascelles, D., Pawson, P., \& Scott, E. M. (2007). Development of the short-form Glasgow Composite Measure Pain Scale (CMPS-SF) and derivation of an analgesic intervention score. Animal Welfare, 16, 97.

Rozanski, E. A., \& Rush, J. E. (2009). Manual colorido de medicina de urgência e terapia intensiva em pequenos animais. Artes Médicas.

Spinosa, H. S., Górniak, S. L., \& Bernardi, M. M. (2002). Farmacologia aplicada à medicina veterinária. Guanabara Koogan.

Viana, F. A. B. (2014). Guia terapêutico veterinário. In Lagoa Santa.

Recebido: 7 de abril, 2020 .

Aprovado: 18 de maio, 2020.

Disponível online: 10 julho, 2020.
Licenciamento: Este artigo é publicado na modalidade Acesso Aberto sob a licença Creative Commons Atribuição 4.0 (CC-BY 4.0), a qual permite uso irrestrito, distribuição, reprodução em qualquer meio, desde que o autor e a fonte sejam devidamente creditados. 
ANEXO 1 - Escala modificada de Glasgow - Short form of the Glasgow Composite Pain Scale.

\begin{tabular}{|c|c|c|c|c|c|c|c|}
\hline \multicolumn{2}{|c|}{ A. Inspecione o cão no canil. Ele está: } & \multirow{2}{*}{$\frac{\text { Escore: }}{0}$} & \multirow[t]{2}{*}{ Av. 1} & \multirow[t]{2}{*}{ Av. 2} & \multirow[t]{2}{*}{ Av. 3} & \multirow[t]{2}{*}{ Av. 4} & \multirow[t]{2}{*}{ Av. 5} \\
\hline \multirow{4}{*}{ (I) } & Calmo & & & & & & \\
\hline & Latindo ou lamentando & 1 & & & & & \\
\hline & Gemendo & 2 & & & & & \\
\hline & Uivando & 3 & & & & & \\
\hline \multirow{5}{*}{ (II) } & Ignorando a ferida ou região dolorida & 0 & & & & & \\
\hline & Olhando para a ferida & 1 & & & & & \\
\hline & Lambendo a ferida & 2 & & & & & \\
\hline & Esfregando a ferida & 3 & & & & & \\
\hline & Mordendo a ferida & 4 & & & & & \\
\hline \multicolumn{8}{|c|}{ B. Coloque a guia no paciente e o conduza para fora do canil. O Paciente caminha/levanta: } \\
\hline \multirow{5}{*}{ (III) } & Normal & 0 & & & & & \\
\hline & Claudicando & 1 & & & & & \\
\hline & Lento ou relutante & 2 & & & & & \\
\hline & Rígido & 3 & & & & & \\
\hline & Recusa-se a mover & 4 & & & & & \\
\hline \multicolumn{8}{|c|}{ C. Aplique leve pressão ao redor da ferida. Qual a reação? } \\
\hline \multirow{6}{*}{ (IV) } & Nenhuma & 0 & & & & & \\
\hline & Olha ao redor & 1 & & & & & \\
\hline & Recua & 2 & & & & & \\
\hline & Rosna ou protege a área & 3 & & & & & \\
\hline & Morde & 4 & & & & & \\
\hline & Late & 5 & & & & & \\
\hline \multicolumn{8}{|c|}{ D. Em geral. O paciente se apresenta: } \\
\hline \multirow{5}{*}{ (V) } & Feliz e contente/animado & 0 & & & & & \\
\hline & Quieto & 1 & & & & & \\
\hline & Indiferente ou não responsivo ao ambiente & 2 & & & & & \\
\hline & Nervoso ou ansioso ou temeroso & 3 & & & & & \\
\hline & Deprimido ou não responsivo à estimulação & 4 & & & & & \\
\hline \multirow{5}{*}{ (VI) } & Confortável & 0 & & & & & \\
\hline & Instável & 1 & & & & & \\
\hline & Agitado & 2 & & & & & \\
\hline & Encurvado ou tenso & 3 & & & & & \\
\hline & Rígido & 4 & & & & & \\
\hline
\end{tabular}

Fonte: (Fossum, 2014). 